\title{
Observation of phonon-induced magnetic deflagration in manganites
}

\author{
F. Macià, ${ }^{1}$ A. Hernández-Mínguez, ${ }^{1}$ G. Abril,${ }^{1}$ J. M. Hernandez, ${ }^{1}$ A. García-Santiago,,${ }^{1}$ J. Tejada, ${ }^{1}$ F. Parisi, ${ }^{2}$ and \\ P. V. Santos ${ }^{3}$ \\ ${ }^{1}$ Departament de Física Fonamental, Facultat de Física, Universitat de Barcelona, Avinguda Diagonal 647, Planta 4, Edifici nou, \\ 08028 Barcelona, Spain \\ ${ }^{2}$ Departamento de Física, Comisión Nacional de Energía Atómica, and Escuela de Ciencia y Tecnología, UNSAM, \\ Avenida General Paz. 1499, 1650 San Martín, Buenos Aires, Argentina \\ ${ }^{3}$ Paul-Drude-Institut für Festkörperelektronik, Hausvogteiplatz 5-7, 10117 Berlin, Germany
}

(Received 26 June 2007; published 14 November 2007)

\begin{abstract}
Very fast magnetic avalanches in (La, Pr)-based manganites are the signature of a phase transition from an insulating blocked charge-ordered antiferromagnetic state to a charge-delocalized ferromagnetic (CD-FM) state. We report here the experimental observation that this transition does not occur either simultaneously or randomly in the whole sample but there is instead a spatial propagation with a velocity of the order of tens of $\mathrm{m} / \mathrm{s}$. Our results show that avalanches originate from the inside of the sample, move to the outside, and occur at values of the applied magnetic field that depend on the CD-FM fraction in the sample. Moreover, upon application of surface acoustic waves at constant magnetic fields, we are able to trigger avalanches at very well-determined values of the temperature and magnetic field. Due to the interaction with the acoustic waves, the number of isolated ferromagnetic clusters in $\mathrm{La}_{0.225} \mathrm{Pr}_{0.40} \mathrm{Ca}_{0.375} \mathrm{MnO}_{3}$ starts to grow across the entire sample in the same way as if it were a magnetic deflagration.
\end{abstract}

DOI: 10.1103/PhysRevB.76.174424

PACS number(s): 75.10.-b, 45.70.Ht, 75.30.Kz

\section{INTRODUCTION}

The study of the coexistence of the two well segregated charge-delocalized ferromagnetic (CD-FM) and chargeordered antiferromagnetic (CO-AFM) phases in manganites, as well as the influence of the applied magnetic field on the occurrence of the magnetic avalanches accompanying the transition from the CO-AFM phase to the CD-FM phase, has, nowadays, a resurgent interest driven by the unusual (but not uncommon) interplay between the static and dynamic properties of the phase separated (PS) state. ${ }^{1-3}$ Phase separation can appear near the boundary of a first order phase transition (FOPT). ${ }^{4}$ In disorder-free magnetic systems, the FOPT occurs at a sharply defined line in the magnetic fieldtemperature $(H-T)$ space. Quenched impurities can lead, under certain circumstances, to the spread of the local transition temperatures (where local means over length scales of the order of the correlation length), leading to the appearance of clustered states with the consequent rounding of the FOPT. ${ }^{5}$ The phase separation observed in manganites seems to belong to this last class of systems, the PS state being the true equilibrium state of the system in the vicinity of the FOPT (Refs. 6 and 7) and not just the result of supercooling or superheating of the system. It has been shown ${ }^{8}$ that the response of the system within the PS state is governed by a hierarchical cooperative dynamics, with state-dependent energy barriers which diverge as the system approaches equilibrium. This slow growing dynamics, which resembles that of glasslike systems, has given place to the construction of phase diagrams of manganites focused on the dynamic properties of the PS state, with regions of the phase diagrams named as "frozen" or "dynamic" PS (Ref. 8) "strain glass" or "strain liquid," and the determination of state-dependent blocking or freezing temperatures. ${ }^{8,10}$ These kinds of processes are not exclusive of PS manganites but are also ob- served in other systems displaying magnetic FOPT. ${ }^{11,12}$

Direct evidences of micrometer phase coexistence in manganites at intermediate temperatures were obtained through electron microscopy ${ }^{13,14}$ and magnetic force microscopy (MFM). ${ }^{15} \mathrm{~A}$ major goal in the description of the low temperature behavior of the PS manganite $\mathrm{La}_{5 / 8-y} \mathrm{Pr}_{y} \mathrm{Ca}_{3 / 8} \mathrm{MnO}_{3}[\operatorname{LPCM}(y), y=3 / 8]$ was achieved recently by Wu et $a .^{2}$ Using a MFM technique, they were able to "photograph" the isothermal evolution of the system as $H$ varies (a metamagnetic transition) and the evolution at fixed $H$ when $T$ increases (the "glass" transition) at the micrometer scale, giving direct evidence of the growing process of the CD-FM phase against the CO-AFM. They conclude, in agreement with the thermodynamic consideration presented in Ref. 10, that the low temperature state accessible after cooling the sample in zero magnetic field could be associated with the supercooled state of the CO-AFM to CD-FM phase transition, the kinetics of the transformation being influenced by accommodation strain between the coexisting phases. The results presented in Ref. 2 were obtained at temperatures above $6 \mathrm{~K}$. Below this temperature, the metamagnetic transition between the frozen CO-AFM state and the equilibrium CD-FM phase does not occur in a continuous way, ${ }^{16,17}$ at least in the time scale of the MFM experiments (seconds), but it occurs sharply at fields above $2 \mathrm{~T}$, presumably in milliseconds.

Experimentally, it has also been shown that the phase separation state is in fact a dynamic process, with the phase fraction of the coexisting CD-FM and CO-AFM states changing continuously as a function of time and temperature, ${ }^{8,9}$ in a fashion that resembles glassy behavior. The glasslike behavior observed at low temperatures is one of the most intriguing features of phase separated manganites. For instance, it was shown that the zero-field low temperature equilibrium state of $\mathrm{La}_{5 / 8-y} \mathrm{Pr}_{y} \mathrm{Ca}_{3 / 8} \mathrm{MnO}_{3}(y=0.4)$ is 
fully CD-FM. ${ }^{10}$ However, this state is not reached after a zero-field cooling (ZFC) process, or even after field cooling under moderated fields. ${ }^{8}$ The distinctive fact is that the state reached in this way is not a conventional metastable supercooled state (where relaxation times decrease with decreasing temperature) but a glasslike state, where the relaxation times increase as temperature is lowered. ${ }^{8,11}$ This behavior is compatible with a free energy landscape similar to that describing spin glasses, but with a distinctive feature: phase competition rather than magnetic interactions seems to be the reason of the observed behavior. When this phase competition couples with some kind of quenched disorder (for instance, chemical substitution), a complex free energy density map can be obtained in real space coordinates, leading to the formation of inhomogeneous states. ${ }^{3,10}$ Adjacent states are separated by energy barriers $U(H, T, x)$, that, in general, are dependent on magnetic field $H$, temperature $T$, and collective state of the system, characterized by the fraction $x$ of the CD-FM phase. ${ }^{8}$ The energy barriers, which are mainly associated to structural differences between the coexisting phases, ${ }^{10}$ imply the existence of a blocking temperature $T_{B}$ $\approx U(H, T, x)$, below which there are no time dependent phenomena for experiments in the time scale of seconds. ${ }^{2,8,10,11}$ Within the blocked region, one of the most intriguing and spectacular experimental facts is observed: the abrupt and fast magnetization jumps occurring at low temperature under the influence of the applied magnetic field. ${ }^{8,16,17}$ These jumps are associated with very fast transitions between the antiferromagnetic and the stable metallic ferromagnetic state and are also accompanied by colossal variations in the electrical resistance of the sample. The ignition of the avalanches is a stochastic process that can be induced by either increasing the applied magnetic field or by waiting long enough at a fixed $H .{ }^{16}$

In this work, we present the study of the dynamics of abrupt phase transition from the CO-AFM to the CD-FM state in $\operatorname{LPCM}(0.4)$ below $6 \mathrm{~K}$ and its spatial evolution. We also introduce in this paper a method to ignite magnetic jumps at well-determined values of both the concentration of the ferromagnetic phase and the applied magnetic field by means of surface acoustic waves (SAWs). The idea of using SAWs to ignite the magnetic avalanches was motivated by the results obtained in molecular magnets where the phenomenon of deflagration ${ }^{18}$ assisted by quantum tunneling ${ }^{19}$ was discovered. A polycrystalline sample of composition $\mathrm{La}_{0.225} \mathrm{Pr}_{0.4} \mathrm{Ca}_{0.375} \mathrm{MnO}_{3}$ and dimensions $2 \times 2 \times 5 \mathrm{~mm}^{3}$ has been used. All the experiments described here were performed by placing the polycrystal inside a commercial magnetometer which can apply magnetic fields up to $5 \mathrm{~T}$ with a sweeping rate of $300 \mathrm{Oe} / \mathrm{s}$, with the longest dimension of the sample parallel to the direction of the external magnetic field.

The article is organized as follows. In the next section (Sec. II), we show the previous magnetic characterization performed on the sample. In Sec. III, the study of the spatial propagation of the abrupt transition along the sample is presented, whereas in Sec. IV, we show the results when the abrupt phase transition is ignited by SAWs. In Sec. V, we discuss the results exposed and, finally, we summarize and conclude our study in Sec. VI.

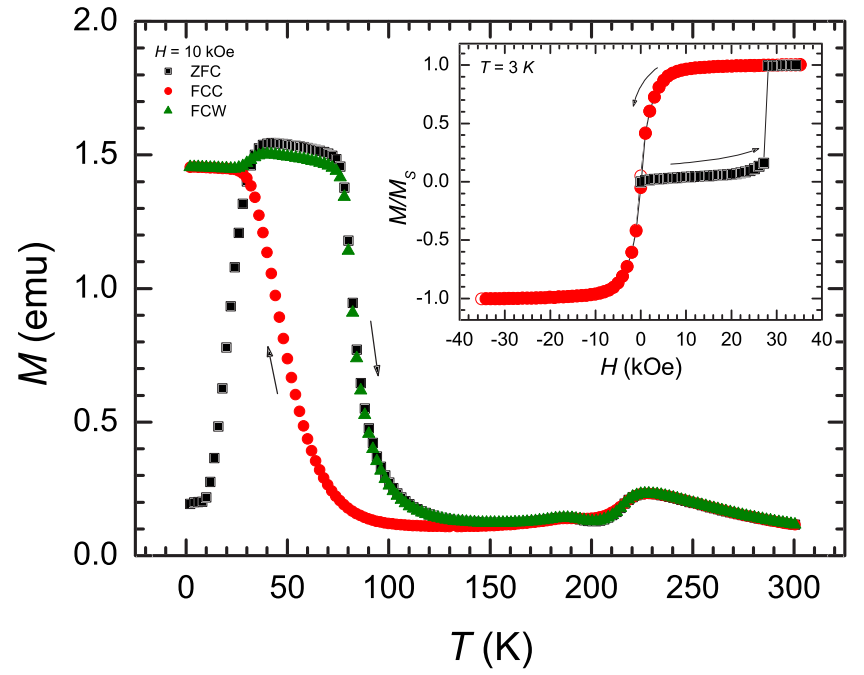

FIG. 1. (Color online) Temperature dependence of the zero-field cooled (squares), field cooled on cooling (circles), and field cooled on warming (triangles) magnetizations of $\mathrm{La}_{0.225} \mathrm{Pr}_{0.4} \mathrm{Ca}_{0.375} \mathrm{MnO}_{3}$, measured for a magnetic field of $10 \mathrm{kOe}$. The inset shows the field dependence of the magnetization with respect to the saturation value, $M_{s}$, at $3 \mathrm{~K}$. The squares correspond to the first magnetization curve, whereas the circles show the magnetic measurements from 35 to $-35 \mathrm{kOe}$ and back to $35 \mathrm{kOe}$.

\section{SAMPLE CHARACTERIZATION}

The sample was first characterized by measuring the temperature and magnetic field dependences of the magnetization. Figure 1 shows that, as $T$ decreases, the sample experiences a transition from a paramagnetic to a charge-ordered state at $T_{\mathrm{CO}}=220 \mathrm{~K}$. A short peak at $190 \mathrm{~K}$ reveals the formation of the CO-AFM state (we cannot distinguish in our experiments between the CO-AFM phase and a charge disordered insulating phase, which can be stabilized by strain at the interphase between the CD-FM and CO-AFM phases ${ }^{21}$ ). A clear ferromagnetic transition appears at a lower temperature $\left(T_{C}=70 \mathrm{~K}\right.$ on cooling, $T_{C}=90 \mathrm{~K}$ on warming). Finally, at $T_{B}=25 \mathrm{~K}$, the blocking temperature associated with the energy barriers between the two phases is clearly seen in the ZFC curve. The inset in Fig. 1 shows the isothermal magnetization curve measured at $3 \mathrm{~K}$. At the first magnetization curve (squares), a magnetic avalanche was detected at $28 \mathrm{kOe}$, the final magnetization being the saturation value, $M_{s}$, which corresponds to the case of having only the ferromagnetic phase. The value of $x$ throughout the first magnetization curve can be estimated at each temperature and magnetic field from the ratio between $M$ and $M_{s}$. This definition is no longer valid once $M_{S}$ has been achieved due to the fact that, from then on, the entire sample is in the CD-FM state as long as it is not heated and zero-field cooled again. We then kept the temperature constant and measured immediately after a $M(H)$ going from $35 \mathrm{kOe}$ down to $-35 \mathrm{kOe}$ and back to $35 \mathrm{kOe}$ (circles). This hysteresis cycle shows that the ferromagnetic state has neither remanence nor coercivity and, consequently, there are no time dependent phenomena. This point has been further verified by measuring the constancy of the magnetization after fast changes of the magnetic field. 


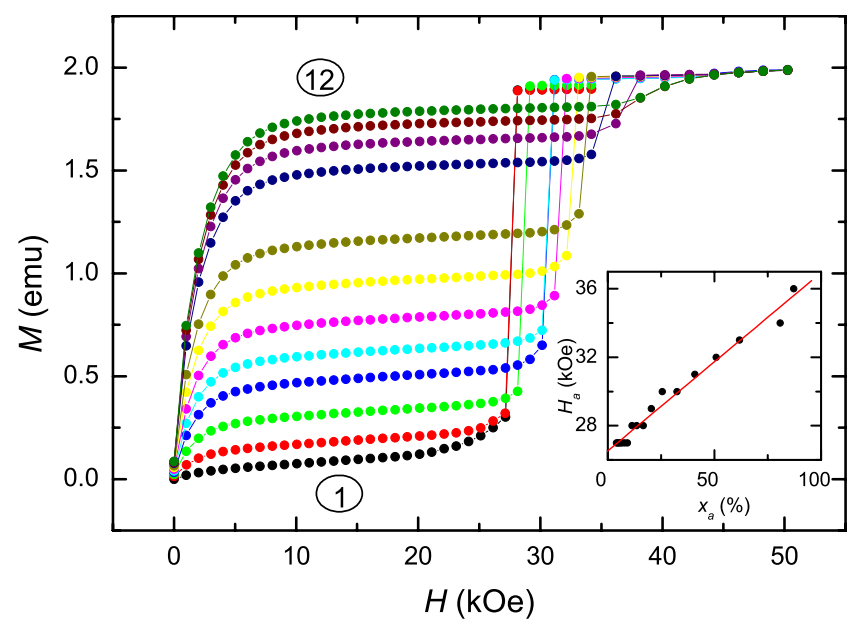

FIG. 2. (Color online) Isothermal magnetization curves at $T$ $=2.5 \mathrm{~K}$ after field cooling the sample. Curve 1 corresponds to the zero-field process whereas curve 12 corresponds to the case when the sample is cooled under $H_{\mathrm{FC}}=22 \mathrm{kOe}$. The inset shows the magnetic field $H_{a}$ at which avalanches occur spontaneously as a function of the ferromagnetic fraction $x_{a}$.

All these results are in full agreement with those previously reported for similar PS manganites. ${ }^{16}$

Figure 2 shows isothermal $M(H)$ curves obtained after cooling the sample down to $T=2.5 \mathrm{~K}$ under different magnetic fields $H_{\mathrm{FC}}$ ranging from $H_{\mathrm{FC}}=0 \mathrm{kOe}$ (curve 1) to $H_{\mathrm{FC}}$ $=22 \mathrm{kOe}$ (curve 12) with an interval of $\Delta H_{\mathrm{FC}}=2 \mathrm{kOe}$. The cooling rate is another important parameter involved in the growing of the CD-FM phase. For this experiment, we keep the cooling rate constant at $10 \mathrm{~K} / \mathrm{min}$. It is clear from Fig. 2 that, at fixed $T$, both the applied magnetic field $H_{a}$ and the fraction of ferromagnetic phase when the avalanche does start, $x_{a}$, depend on $H_{\mathrm{FC}}$. In addition, $H_{a}$ is a well-determined field value that grows linearly on $x_{a}$, as can be seen at the inset of Fig. 2. Finally, the magnetization after the magnetic avalanche always reaches the value corresponding to the saturation magnetization of the ferromagnetic phase, $x=1$. The different curves $M(H)$ of Fig. 2 correspond, therefore, to different initial blocked states, tuned by the cooling field. Once the avalanche is ignited, the CD-FM phase starts to grow against the CO-AFM phase, resulting in heat that further accelerates the phase transition process. ${ }^{16}$ The propagation through the sample of this spin reversing process is dominated, therefore, by the rate between the heat delivered and the heat conduction through the sample. Both processes are very sensitive to the phase fraction $x_{a}$ at the beginning of the avalanche. ${ }^{16}$

In Fig. 3, we show isothermal $M(H)$ curves without avalanches registered at several temperatures comprised between 3 and $5 \mathrm{~K}$ after zero-field cooling the sample. Magnetic avalanches are turned on due to the interplay between the local increase of the CD-FM fraction and the heat released in this microscopic transformation. ${ }^{16}$ Therefore, to avoid the ignition of these avalanches, we increased the number of points that were measured at the same interval of the applied magnetic field with respect to the hysteresis curve at the inset of Fig. 1. As each point takes several sec-

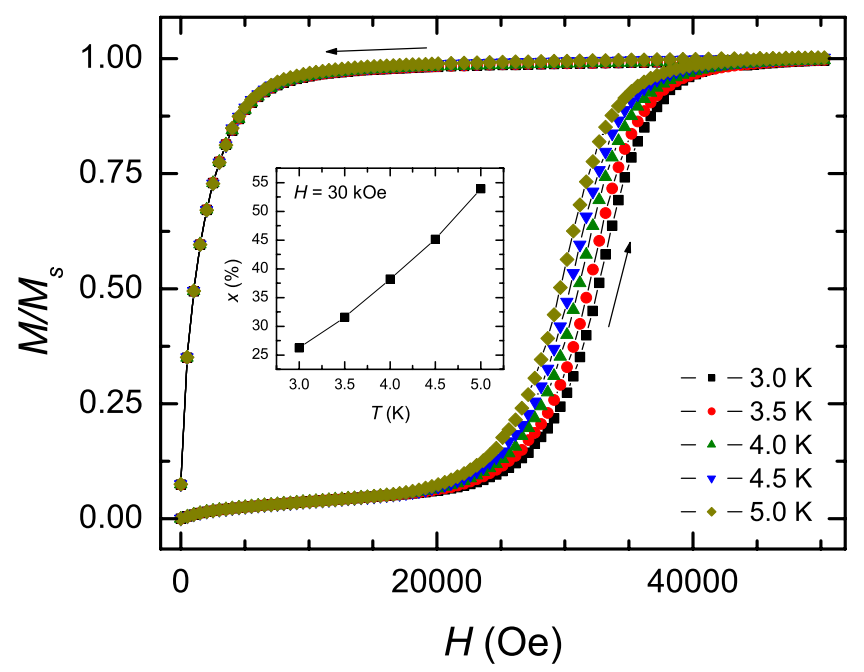

FIG. 3. (Color online) Isothermal magnetization curves registered at several temperatures between 3 and $5 \mathrm{~K}$ after zero-field cooling the sample. The inset shows the temperature dependence of the ferromagnetic phase fraction at the first magnetization curve, $x$, for an applied magnetic field of $30 \mathrm{kOe}$.

onds to be measured, this reduces the average sweep rate of the applied magnetic field, allowing the sample to thermalize and making it possible to register the $M$ values of the PS state at many magnetic fields and temperatures where otherwise the sample would have already transited to the CD-FM state. The data of Fig. 3 show that first magnetization curves for field values smaller than $20 \mathrm{kOe}$ coincide, indicating that the FM phase fraction $x$ is nearly the same at all temperatures between 3 and $5 \mathrm{~K}$ for $H$ values lower than $20 \mathrm{kOe}$. According to the definition of $x$ mentioned above, a value of $x$ smaller than $8 \%$ was estimated at $20 \mathrm{kOe}$. For magnetic fields larger than $20 \mathrm{kOe}$, there is, however, a separation between the different first magnetization curves indicating that the accessible CD-FM phase fraction depends on both the temperature and the magnetic field. Time dependent phenomena occur in this regime of values of $T$ and $H$ indicating that the phase separation is a dynamic process which may be characterized by a barrier height $U(H, T, x)$ that separates the $\mathrm{CD}-\mathrm{FM}$ and the CO-AFM states. The changes in $x$ are therefore due to thermal transitions above this barrier height. The temperature dependence of $x$ for $30 \mathrm{kOe}$ is given in the inset of Fig. 3. These values of $x$ correspond, therefore, to the blocked phase fraction compatible with the time scale of the experiment, which in this case is of the order of seconds.

\section{SPATIAL PROPAGATION OF THE ABRUPT TRANSITION}

\section{A. Experimental setup}

In the next section, we will describe the experiments and results when local magnetic measurements are used to study the magnetic avalanches. ${ }^{20}$ Figure 4(a) shows the experimental setup. The sample was placed inside a plastic tube and three coils of four turns each were wound around the tube, one in the center (coil B) and two at the two edges of the 


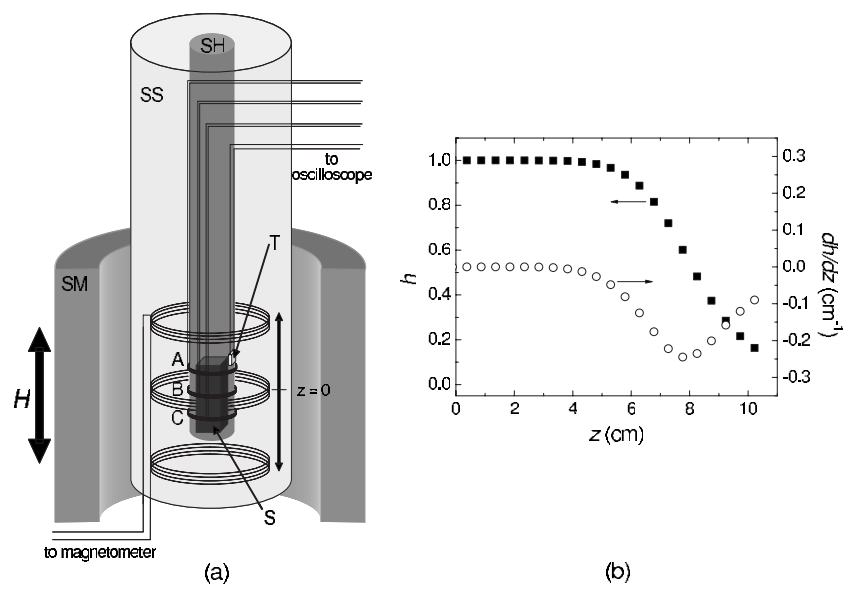

FIG. 4. (a) Experimental setup. The sample (S) is mounted on a sample holder $(\mathrm{SH})$ with three detection coils (A, B, and C) and one thermometer $(\mathrm{T})$. This ensemble is introduced in the sample space (SS) of a commercial magnetometer and centered in the superconducting magnet (SM) that applies the magnetic field. The center of the SM is taken as $z=0$. (b) Reduced applied magnetic field (solid squares), $h(z)=H(z) / H_{\max }\left[H_{\max }=H(z=0)\right]$, and the numerical derivative of such magnitude (open circles), $d h / d z(z)$.

sample (coils A and C, respectively). In Fig. 4(b), we show the spatial dependence of the reduced magnetic field, $h$ $=H(z) / H_{\max }$, defined as the ratio between the applied magnetic field value at the position $z, H(z)$, and the nominal value of the magnetic field applied at the center of the superconducting magnet, $H_{\max }$. By placing the sample in different positions along the $z$ axis of the cryostat, we may change the field gradient acting along one of the dimensions, as it can be seen in Fig. 4(b) from the curve of the numerical derivative of the reduced magnetic field, $d h / d z$. In these experiments, we placed the sample so that the so-called $z$ direction corresponds to the longest dimension of the sample [see Fig. $4(\mathrm{a})]$.

\section{B. Results}

Figure 5 shows the voltages detected by the three coils, which are proportional to the variation of magnetization as a function of time, $d M / d t$. $t=0$ corresponds to the instant when the avalanche does start and so the three coils detect a nonzero voltage value. These experiments were performed at temperatures comprised between 3 and $5 \mathrm{~K}$, when the sample was centered at $z=0$. As we could only monitorize two signals simultaneously, we repeated the experiment at each temperature several times, registering two different coils each time. The results turned to be highly reproducible and deterministic in this temperature range, so we used the signal detected by coil B to synchronize the signals of all three coils. From Fig. 5, we conclude that the ignition process of the avalanche occurs systematically in the interior of the sample and then moves to the outside. This is due to the fact that the middle of the sample is the place where thermal dissipation is worst. As the extremes of the sample are in thermal contact with the cool helium gas, the heat released

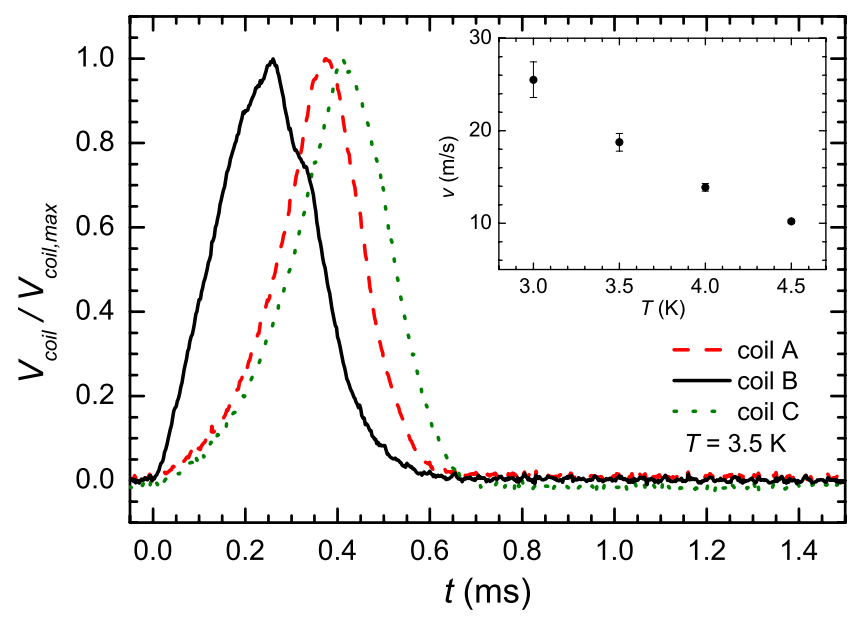

FIG. 5. (Color online) Time variation of the magnetization as detected by coils A (dashed line), B (solid line), and C (dotted line) at $3.5 \mathrm{~K}$. These three signals have been normalized with respect to their maximum values. The inset shows the propagation velocity of the phase transformation front along the sample as a function of temperature.

by the transformation of CO-AFM metastable regions to CD-FM ones is better thermalized at the edges than at the center. Therefore, when the applied magnetic field is homogeneous along the sample, the local temperature increase necessary to ignite the avalanche is more easily achieved in the inner regions of the sample.

The velocity of propagation of the transformation front can be roughly estimated as $v=L / \Delta t$, where $L$ is the length of the sample, $\Delta t$ is defined as $\Delta t=\left(t_{A}-t_{B}\right)+\left(t_{C}-t_{B}\right)$, and $t_{A}$, $t_{B}$, and $t_{C}$ correspond to the times when the signal detected, respectively, by coils $\mathrm{A}, \mathrm{B}$, and $\mathrm{C}$ is maximum. The thermal dependence of this velocity is shown in the inset of Fig. 5, according to which the average value of $v$ is about $20 \mathrm{~m} / \mathrm{s}$ at $3.5 \mathrm{~K}$. As $T$ and $x$ are strongly correlated, the velocity of the avalanche also depends on the value of $x$ when the abrupt phase transition is ignited.

The results shown in Fig. 5 correspond to the case when the applied magnetic field is uniform along the sample. We have also performed experiments shifting the position of the sample along the $z$ axis. In these cases, the sample suffers the effect of the field and its gradient in such a way that we can obtain variations of the magnetic field along the longest dimension up to $10 \%$ of the maximum value of $H$ applied. In this case, we have used two new detection coils, named 1 and 2, located at the positions where coils $\mathrm{A}$ and $\mathrm{C}$ previously were. Figure 6 shows the voltages detected by these coils as a function of time for three different positions along the $z$ direction. Considering that the variation of the magnetic field between the two edges of the polycrystal can be roughly estimated as $\Delta H \sim H_{\max }(d h / d z) L, H_{\max } \sim 28 \mathrm{kOe}$, and taking $d h / d z$ from Fig. 4(b), values of $\Delta H \sim 100,800$, and 2000 Oe can be obtained at $z=4.0,5.5$, and $6.5 \mathrm{~cm}$. The time sequence of the peaks measured by the detection coils at Fig. 6 shows that the presence of a field gradient dramatically affects the origin and propagation of the abrupt phase transition along the sample. This is more clearly seen in Fig. 7, 


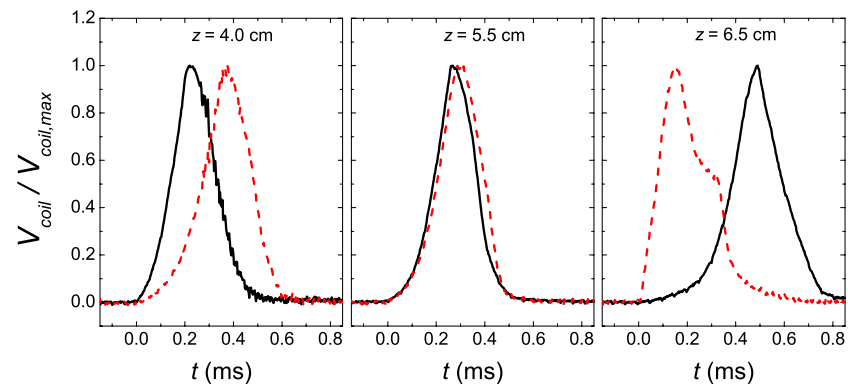

FIG. 6. (Color online) Time evolution of the voltages detected by coils 1 (solid line) and 2 (dashed line) for different locations of the sample. From left to right, $z=4.0 \mathrm{~cm}$ (left panel), $z=5.5 \mathrm{~cm}$ (central panel), and $z=6.5 \mathrm{~cm}$ (right panel). All signals have been normalized to their maximum values. As $z$ goes up and the gradient of the applied magnetic field increases, the time sequence of the signal registered by the two detection coils is reversed. The experiment was performed at $3 \mathrm{~K}$.

where we have plotted the time difference between the maxima of the signals in the two coils, $t_{1}-t_{2}$, as a function of $z$. The change in this magnitude reflects that the place of the sample where the abrupt phase transition starts can be tuned through the strength of the field gradient, a fact that is consistent with a spatial dependence of the distribution of energy barriers between the CO-AFM and CD-FM phases.

\section{PHONON-INDUCED AVALANCHES}

\section{A. Experimental setup}

The magnetoacoustic experiments were performed by using interdigital transducers ${ }^{22}$ (IDTs) deposited on $128 X Y$-cut $\mathrm{LiNbO}_{3}$ substrates with dimensions of $2 \times 6 \times 1 \mathrm{~mm}^{3}$ and a manganite sample directly glued onto the piezoelectric, see Fig. 8(a). To monitorize the temperature of the sample a thermometer was attached to the manganite (not shown in the figure). This setup was placed inside the commercial magnetometer and cooled down to temperatures between 2

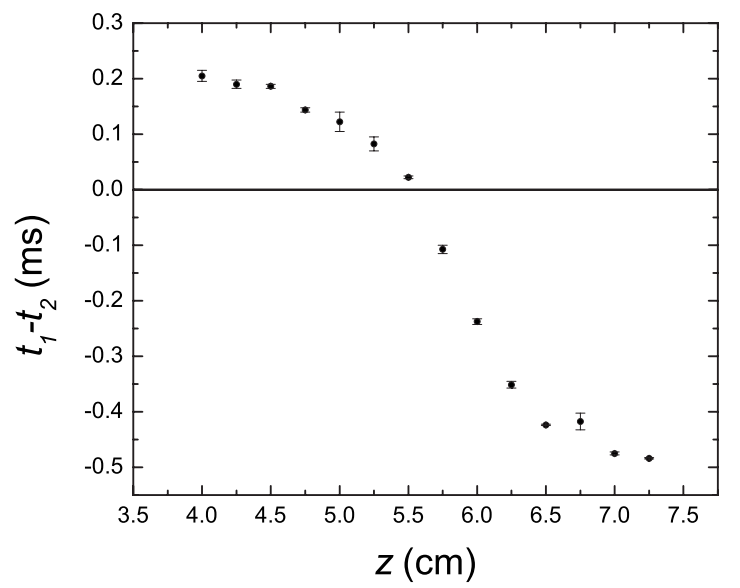

FIG. 7. Variation of the time difference between the maxima of the signal of the two coils, $t_{1}-t_{2}$, with respect to $z$. The avalanches were ignited at $3.5 \mathrm{~K}$.

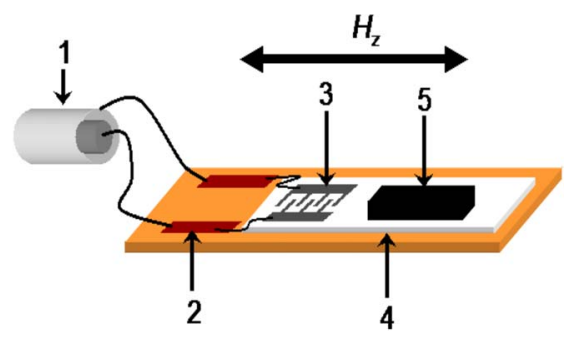

(a)

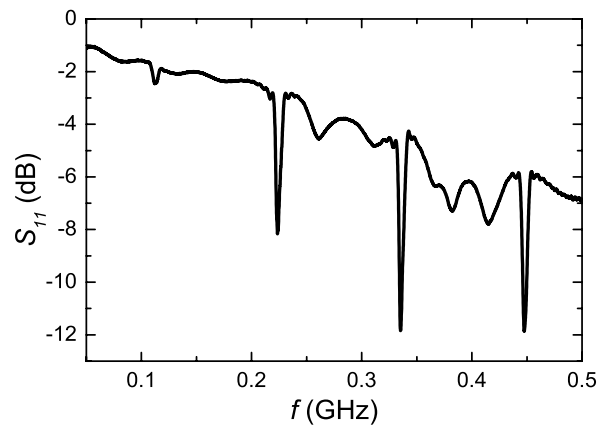

(b)

FIG. 8. (Color online) (a) Experimental setup. 1, coaxial cable; 2 , conducting stripes; 3 , interdigital transducers (IDT) $; 4, \mathrm{LiNbO}_{3}$ substrate; 5, manganite sample. The longest dimension of the sample is oriented parallel to the applied magnetic field $H_{z}$. (b) Frequency dependence of the radio-frequency reflection coefficient $S_{11}$ of the acoustic transducer (IDT) measured at $T=10 \mathrm{~K}$ under a nominal rf power of $10 \mathrm{dBm}$.

and $6 \mathrm{~K}$. The microwaves for the SAW generation were transported to the transducers by coaxial cables. The frequency dependence of the reflection coefficient $S_{11}$ of the IDTs, see Fig. 8(b), clearly showed that the resonant frequencies of the SAWs are multiple harmonics of the fundamental frequency of $112 \mathrm{MHz}$, accordingly with results previously published. ${ }^{23}$

\section{B. Results}

To study the magnetic avalanches induced by SAWs, the sample was first field cooled down to the desired temperature under different applied magnetic fields in order to obtain different values of $x$ as starting points. The magnetic field was then swept at a constant rate of $300 \mathrm{Oe} / \mathrm{s}$ up to a predefined value $H$ lower than $H_{a}$ (otherwise, the avalanche would have been ignited spontaneously while the applied magnetic field was changing). A rectangular microwave pulse with well-controlled frequency $(f=224 \mathrm{MHz})$, intensity $(10 \mathrm{dBm})$, and duration (1 s) was then applied to trigger the magnetic avalanches. As the applied magnetic field remained constant and the triggering dynamics is well defined, it became possible to carry out fast magnetic measurements (time resolution of $10 \mu \mathrm{s}$ ) by continuously recording the voltage detected by the rf superconducting quantum interference device of the magnetometer.

Figure 9 shows the time evolution of the magnetization and temperature of an avalanche ignited at an initial tempera- 


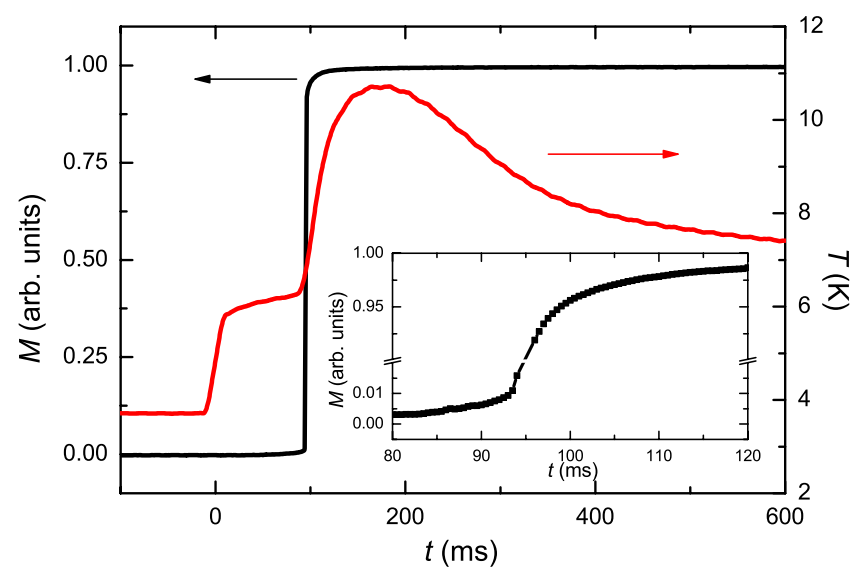

FIG. 9. (Color online) Time evolution of magnetization and temperature of the sample during the avalanche process at $T=4 \mathrm{~K}$. The avalanche was ignited by a SAW pulse applied at $t=0$ under $H=28 \mathrm{kOe}$ in a sample that was cooled at $H_{\mathrm{FC}}=11 \mathrm{kOe}$. The inset shows $M(t)$ over a narrower time window. It is clear that the biggest magnetization change occurs in only a few milliseconds.

ture $T=4 \mathrm{~K}$ and $H=28 \mathrm{kOe}$ by a SAW pulse applied at $t$ $=0$. The magnetic avalanche takes tens of milliseconds to be ignited and, during that time, the magnetization hardly changes. Nevertheless, the temperature increases within nanoseconds after the onset of the SAW pulse and, afterward, the ignition of the magnetic avalanche is accompanied by a second, more pronounced increase in temperature. After the magnetization has changed, the manganite needs near $1 \mathrm{~s}$ to return to the thermodynamic equilibrium temperature which is higher than $4 \mathrm{~K}$ (about $6 \mathrm{~K}$ in our case) because the SAW pulse is still being applied.

Figure 10 shows the time evolution of the magnetization for avalanches induced at the same $T$ and $H_{\mathrm{FC}}$ but different applied magnetic fields. As we have observed, the avalanches correspond to the motion of a magnetization front of ferromagnetic clusters from the interior to the exterior of the sample. Since the magnetization change has been measured with a resolution time of $10 \mu \mathrm{s}$, it has been possible to detect

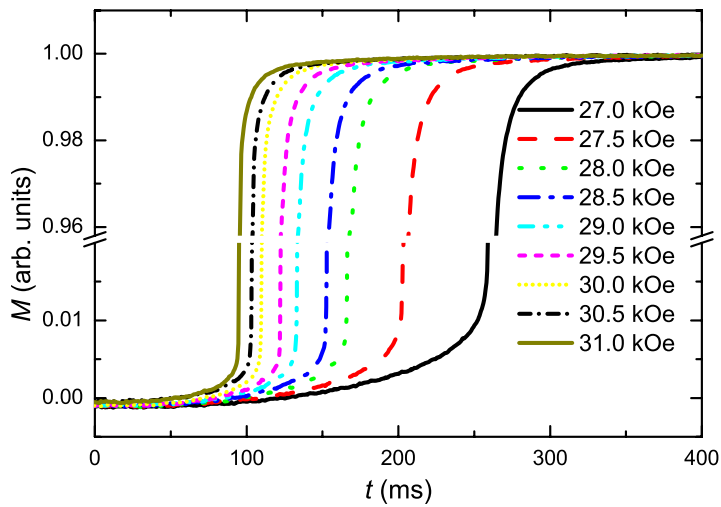

FIG. 10. (Color online) Magnetic avalanches ignited by SAWs at $T=3$ under different applied magnetic fields. In all cases, the manganite was cooled down under a magnetic field of $H_{\mathrm{FC}}$ $=13 \mathrm{kOe}$.
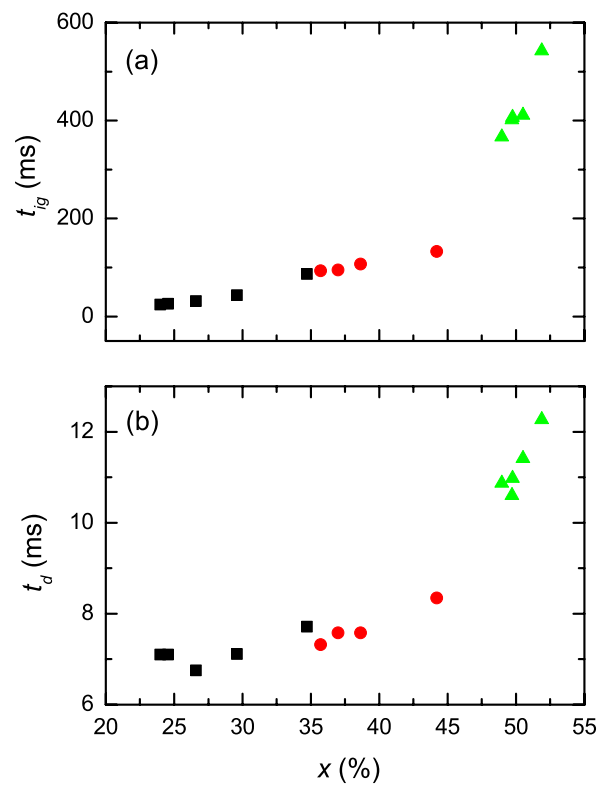

FIG. 11. (Color online) Dependence of the (a) ignition time $t_{\mathrm{ig}}$ and (b) deflagration time $t_{d}$ on the CD-FM phase fraction $x$ just before the SAW pulse is applied. The squares correspond to $T$ $=2 \mathrm{~K}$, circles to $T=3 \mathrm{~K}$, and triangles to $T=4 \mathrm{~K}$. Measurements were performed at $H=28 \mathrm{kOe}$.

two main stages during the magnetization reversal process. The first stage is the ignition stage, characterized by the time interval, $t_{\mathrm{ig}}$, between the beginning of the SAW pulse and the formation of a stable deflagration front (the "flame") propagating through the sample. The second stage corresponds to the so-called magnetic deflagration process, which is also characterized by a deflagration time $t_{d}$ and the local temperature of the flame, $T_{f}$, which is much higher than the one detected by the thermometer. The heat energy released in the flame during the abrupt phase transition corresponds to the difference between the free energy of the metastable COAFM and the stable CD-FM states plus the Zeeman energy $\Delta E=g \mu_{B} H \Delta S$ per spin of the ferromagnetic clusters. By neglecting heat dissipation to the surroundings, the final temperature measured by the thermometer at Fig. 9 becomes a good estimate for the temperature of the magnetic "ashes," which in our case consist of ferromagnetic clusters with magnetic moments aligned with the magnetic field.

\section{DISCUSSION}

Figures 11(a) and 12(a) [Figs. 11(b) and 12(b)] show the dependence of $t_{\mathrm{ig}}\left(t_{d}\right)$ on the concentration $x$ and on the magnetic field $H$ at which SAW-induced avalanches have been ignited, respectively. To explain these data in terms of magnetic deflagration, let us start by considering the dependence of the velocity of the flame on its temperature and magnetic field, ${ }^{18}$

$$
v=\sqrt{\frac{\kappa}{\tau}}=\sqrt{\frac{\kappa}{\tau_{0}}} \exp \left[\frac{-U(H, T, x)}{2 k_{B} T_{f}}\right],
$$

where $\kappa$ is the thermal diffusivity, $U(H, T, x)$ is the barrier height, $\tau_{0}$ is the characteristic attempt frequency, and $T_{f}$ is 

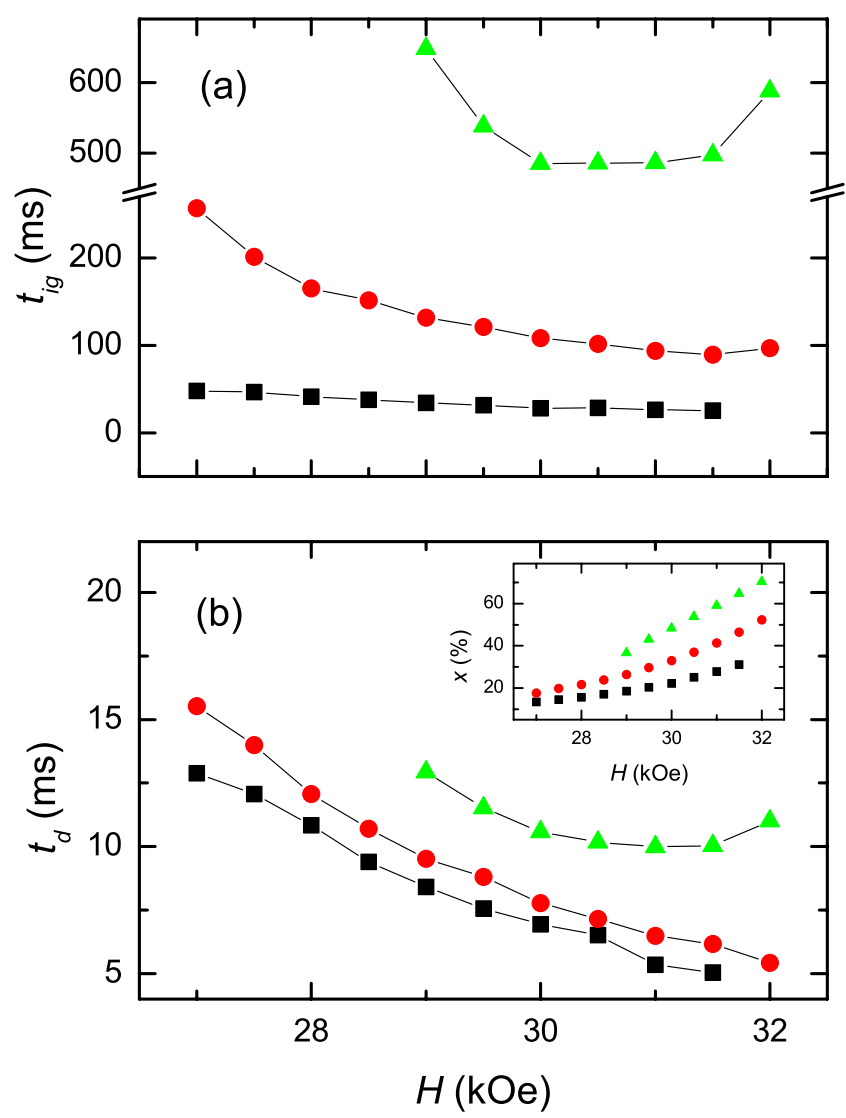

FIG. 12. (Color online) Magnetic field dependence of (a) $t_{\text {ig }}$ and (b) $t_{d}$. The squares correspond to $T=2 \mathrm{~K}$, circles to $T=3 \mathrm{~K}$, and triangles to $T=4 \mathrm{~K}$. The inset shows the CD-FM phase fraction $x$ just before the SAW pulse is applied at different magnetic fields.

the temperature of the flame, which is also a function of $x$ and $H$. The dependence of $v \propto 1 / t_{d}$ with the initial temperature of the experiment enters mainly through $x$. When the fraction of the (blocked) ferromagnetic phase increases, the barrier $U(H, T, x)$ increases and, consequently, a longer time $t_{\text {ig }}$ is necessary to ignite. Moreover, the temperature and, consequently, the velocity of the flame will also decrease exponentially with $x,{ }^{8}$ in agreement with the result shown in Fig. 11(b).

The variation of $T_{f}$ when changing the magnetic field depends on both $H$ and $x$ : as $x$ increases with $H$ and $T$, a nonmonotonous behavior is expected for $t_{\text {ig }}$ and $t_{d}$ as a function of $H$. The data shown in Fig. 12(b) indicate that $t_{d}$ decreases with $H$ for temperatures below $4 \mathrm{~K}$. This experimental result correlates well with the fact that in this temperature range, the strongest effect on $v$ is due to the reduction of the barrier height with the applied magnetic field. At $T=4 \mathrm{~K}$, the competition between the reduction in both $U(H, T, x)$ and $T_{f}$ could be the reason why $t_{d}$ shows an inflection in its curvature around $H=3.1 \mathrm{~T}$. The dependence of $t_{\text {ig }}$ on $H$ does also show a decrease for field values lower than $H=3.1 \mathrm{~T}$. For fields larger than $H=3.1 \mathrm{~T}$ and at $T=4 \mathrm{~K}$, the ignition process is slower as a consequence of the decreasing of the heat release associated with the higher values of $x$.

\section{CONCLUSION}

In conclusion, we have demonstrated that the magnetic avalanches associated with the transition from the CO-AFM metastable state into the CD-FM metallic state by the influence of the applied magnetic field correspond to a phase transformation mechanism that moves along the sample, being ignited in the inside and propagating to the edges. The fact that the grain boundaries do not trap the phase propagation agrees very well with the deflagration phenomena in which the propagation is mediated by heat. This mechanism reflects, at macroscopic scales, the same physics shown ${ }^{2}$ at microscopic ones: when the applied field overcomes a certain temperature and state-dependent threshold value, the system becomes unblocked, inducing the growth of the CD-FM phase against the unstable CO-AFM phase. We have also shown that by applying a magnetic field gradient, it is possible to change the place in the sample where this threshold is overcome, allowing to modify the position where the avalanche is ignited. We have also shown the generation of the magnetic avalanches in PS perovskites induced by surface acoustic waves. The quasideterministic magnetization ignition provided by the SAWs allows us to investigate the dynamics of the magnetic avalanches, which move through the sample like a deflagration front. We have, therefore, defined and measured both the ignition time of the deflagration and the speed of the flame, as well as their dependences on the applied magnetic field and on the ferromagnetic phase fraction.

\section{ACKNOWLEDGMENTS}

F.M. and A.H.-M. thank the Spanish Ministerio de Educación y Ciencia for a research grant. J.M.H. thanks the Ministerio de Educación y Ciencia and the Universitat de Barcelona for a Ramón y Cajal research contract. A.G.-S. thanks Universitat de Barcelona for an associate professor contract. We thank W. Seidel and S. Krauss for the fabrication of the piezoelements and the Acciones Integradas (Spain)/DAAD (Germany) program for financial support. We thank G. Leyva for providing the samples.

\footnotetext{
${ }^{1}$ N. Mathur, Nat. Mater. 5, 849 (2006).

${ }^{2}$ W. Wu, C. Israel, N. Hur, S. Park, S.-W. Cheong, and A. de Lozanne, Nat. Mater. 5, 881 (2006).

${ }^{3}$ E. Dagotto, Science 309, 257 (2005).

${ }^{4}$ E. Dagotto, New J. Phys. 7, 67 (2005).
}

\footnotetext{
${ }^{5}$ Y. Imry and M. Wortis, Phys. Rev. B 19, 3580 (1979).

${ }^{6}$ A. Moreo, M. Mayr, A. Feiguin, S. Yunoki, and E. Dagotto, Phys. Rev. Lett. 84, 5568 (2000).

${ }^{7}$ P. Levy, F. Parisi, L. Granja, E. Indelicato, and G. Polla, Phys. Rev. Lett. 89, 137001 (2002).
} 
${ }^{8}$ L. Ghivelder and F. Parisi, Phys. Rev. B 71, 184425 (2005).

${ }^{9}$ P. A. Sharma, S. B. Kim, T. Y. Koo, S. Guha, and S.-W. Cheong, Phys. Rev. B 71, 224416 (2005).

${ }^{10}$ J. Sacanell, F. Parisi, J. C. P. Campoy, and L. Ghivelder, Phys. Rev. B 73, 014403 (2006).

${ }^{11}$ K. Kumar, A. K. Pramanik, A. Banerjee, P. Chaddah, S. B. Roy, S. Park, C. L. Zhang, and S.-W. Cheong, Phys. Rev. B 73, 184435 (2006).

${ }^{12}$ M. K. Chattopadhyay, S. B. Roy, and P. Chaddah, Phys. Rev. B 72, 180401(R) (2005).

${ }^{13}$ M. Uehara, S. Mori, C. Chen, and S.-W. Cheong, Nature (London) 399, 560 (1999).

${ }^{14}$ Y. Murakami, J. H. Yoo, D. Shindo, T. Atou, and M. Kikuchi, Nature (London) 423, 965 (2003).

${ }^{15}$ L. Zhang, C. Israel, A. Biswas, R. L. Greene, and A. de Lozanne, Science 298, 805 (2003).

${ }^{16}$ L. Ghivelder, R. S. Freitas, M. G. das Virgens, M. A. Continentino, H. Martinho, L. Granja, M. Quintero, G. Leyva, P. Levy, and F. Parisi, Phys. Rev. B 69, 214414 (2004).

${ }^{17}$ R. Mahendiran, A. Maignan, S. Hébert, C. Martin, M. Hervieu, B. Raveau, J. F. Mitchell, and P. Schiffer, Phys. Rev. Lett. 89, 286602 (2002).

${ }^{18}$ Y. Suzuki et al., Phys. Rev. Lett. 95, 147201 (2005).

${ }^{19}$ A. Hernández-Mínguez, J. M. Hernandez, F. Macià, A. GarcíaSantiago, J. Tejada, and P. V. Santos, Phys. Rev. Lett. 95, 217205 (2005).

${ }^{20}$ A. Hernández-Mínguez, F. Macià, J. M. Hernandez, J. Tejada, L. H. He, and F. F. Wang, Europhys. Lett. 75, 811 (2006).

${ }^{21}$ V. Podzorov, B. G. Kim, V. Kiryukhin, M. E. Gershenson, and S-W. Cheong, Phys. Rev. B 64, 140406(R) (2001).

${ }^{22}$ K. Yamanouchi, C. H. S. Lee, K. Yamamoto, T. Meguro, and H. Odagawa, Proceedings of the 1992 IEEE Ultrasonics Symposium (IEEE, New York, 1992), p. 139.

${ }^{23}$ J. M. Hernandez, P. V. Santos, F. Macià, A. García-Santiago, and J. Tejada, Appl. Phys. Lett. 88, 012503 (2006). 